médecine sciences $1988 ; 7: 106-112$

\title{
La transplantation d'organe comme traitement des maladies métaboliques héréditaires
}

Les progrès considérables réalisés dans les transplantations d'organes en font un possible traitement de nombreuses maladies héréditaires. Le but de la transplantation est, dans ces affections, soit d'introduire un tissu synthétisant l'enzyme absente ou anormale chez le receveur, soit de remplacer un organe dont l'atteinte, secondaire à la maladie, en domine la symptomatologie, soit encore de réaliser ces deux opérations à la fois. Les progrès futurs sont attendus principalement dans deux directions : une amélioration des méthodes destinées à prévoir le rejet de greffons non totalement compatibles, et l'autogreffe de cellules génétiquement recombinées par introduction du gène codant pour la fonction manquante.

Olivier Bernard

\section{Alain Fischer Jean-Pierre Grünfeld}

\section{ADRESSES}

O. Bernard: service d'hépatologie, département de pédiatrie, hôpital de Bicêtre, 94275 Kremlin Bicêtre, France.

A. Fischer: unité d'immunologie et d'hématologie, département de pédiatrie, hôpital des Enfants-Malades, 75743 Paris Cedex 15, France.

J.-P. Grünfeld : département de néphrologie, 406

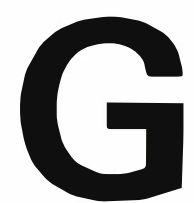

râce à la maîtrise acquise, la transplantation d'organes connaît de nouvelles indications: remplacer un organe déficient n'est qu'une étape ; restaurer une activité enzymatique et guérir ainsi certaines maladies héréditaires du métabolisme, tel est le nouveau champ d'application de la transplantation de certains organes.

En fait, ce propos ambitieux doit être tempéré. Ce type de traitement ne s'applique aujourd'hui qu'à un petit nombre de maladies métaboliques; le défaut enzymatique doit être bien caractérisé (et localisé) ; la surcharge de certains tissus est inaccessible. Dans quelques cas, on se contente de remplacer l'organe détruit dont l'atteinte est la cause (par exemple, la moelle dans l'ostéopétrose) ou la conséquence principale de la maladie métabolique (greffe de rein dans le diabète). Tout différent est l'espoir d'introduire l'activité enzymatique absente avec le transplant et de supprimer ainsi les autres manifestations viscérales de la maladie.

Quatre organes ont été transplantés avec l'espoir, fondé ou non, de corriger une maladie métabolique: le pancréas, le foie, la moelle osseuse et le rein. Plus de 1000 transplantations pancréatiques ont été effectuées dans le monde depuis 1966 chez des malades ayant un diabète insulinodépendant ; souvent une transplantation rénale a été associée car ces malades avaient également une néphropathie diabétique avancée. Les modalités et les indications de la transplantation pancréatique représentent un vaste champ de recherches (voir l'article de P. Vexiau, page 413 de ce numéro). Nous nous limiterons à la transplantation du foie chez l'enfant (où se pose souvent l'indication de corriger une anomalie métabolique grave), à la transplantation de la moelle osseuse et, accessoirement, à celle du rein.

Dans tous les cas se discute aussi le problème de la date de la transplantation par rapport à l'évolution de la maladie.

\section{La transplantation hépatique chez l'enfant}

Dans le Registre Européen de Transplantation hépatique (contenant 1218 malades transplantés dont 
168 enfants), la transplantation était justifiée par une maladie métabolique dans 58 cas, 32 adultes (soit $3 \%$ des greffes) et 26 enfants (soit 15,5\% des greffes hépatiques à cet âge) [1]. Les remarquables progrès de la transplantation hépatique au cours des dernières années laissent espérer qu'au moins $70 \%$ des enfants opérés actuellement seront en vie cinq ans après la greffe [2]

- Maladies létales par atteinte directe du foie. La plupart des transplantations hépatiques faites dans ces cas concernent des maladies héréditaires exposant à une insuffisance hépatocellulaire, à une cirrhose ou à un risque de carcinome hépatique, et pour lesquelles il n'existe pas actuellement d'autre traitement efficace.

- Le déficit en $\alpha$-1-antitrypsine ( $\alpha$-1AT) est une maladie à transmission autosomique récessive. L' $\alpha$-l-AT est une glycoprotéine qui peut inhiber plusieurs enzymes protéolytiques; elle est synthétisée principalement par l'hépatocyte. Chez les sujets homozygotes pour la mutation ponctuelle Z, la concentration de l' $\alpha$ 1-AT dans le sérum est très basse et ces sujets sont exposés à une maladie hépatique survenant dans l'enfance et à un emphysème pulmonaire obstructif pouvant survenir à l'âge adulte ; $17 \%$ des homozygotes $Z$ présentent des signes d'atteinte hépatique pendant l'enfance; la maladie hépatique évolue dans un cas sur deux vers une cirrhose et vers la mort à un âge variable. La transplantation hépatique est le seul traitement possible de ces cirrhoses [3] ; elle permet de confirmer que le foie est la principale source de l' $\alpha$-l-AT du sérum puisque la concentration sérique d' $\alpha-1-A T$ revient à la normale après la transplantation: le phénotype de l' $\alpha$-l-AT du donneur devient détectable dans le sérum du receveur dès le premier jour suivant la transplantation, alors que le phénotype du receveur n'est plus détectable au plus tard trois jours après la transplantation [4]. Il reste à montrer que la modification de la concentration et du phénotype de l' $\alpha$-l-AT sérique permettra, comme on peut l'espérer, de prévenir l'emphysème pulmonaire à l'âge adulte.

- La tyrosinémie héréditaire de transmission récessive autosomique, se $\mathrm{m} / \mathrm{s} n^{\circ} 7$ vol. 4 , septembre 88 révèle chez les nourrissons soit comme une insuffisance hépatocellulaire aiguë rapidement mortelle au cours des premiers mois de la vie, soit comme une cirrhose souvent associée à un rachitisme, dans la deuxième moitié de la première année, et expose à un risque de carcinome hépato-cellulaire. Outre les signes d'atteinte directe du foie, cette maladie est particulière par une augmentation importante de la concentration de tyrosine et de méthionine dans le sang et par une augmentation remarquable de la concentration sérique de l' $\alpha$-foetoprotéine. L'enzyme déficitaire, la fumaryl acéto-acétate hydrolase, est surtout présente dans le foie et les reins. Le déficit entraîne une accumulation d'un métabolite, la succinyl acétone, dont le dosage dañs les urines permet le diagnostic. La transplantation hépatique est le seul traitement de cette maladie qui est spontanément toujours mortelle [5]. Après transplantation, les signes de la maladie, et en particulier l'augmentation de l' $\alpha$-fœetoprotéine, disparaissent et le métabolisme hépatique de la tyrosine redevient normal. Cependant, chez quelques malades, une excrétion urinaire excessive de succinyl acétone persiste, en raison du déficit enzymatique rénal [6].

- Le mécanisme moléculaire de la maladie de Wilson, également transmise sur un mode récessif autosomique, n'est pas encore clairement connu: il associe sans doute un défaut de synthèse par les hépatocytes de la céruloplasmine, protéine transportant le cuivre dans le plasma, et un défaut d'excrétion du cuivre dans la bile. L'accumulation de cuivre dans le foie est responsable d'une maladie hépatique débutant dans l'enfance et dont la gravité de l'expression clinique est variable, allant d'une simple augmentation chronique des transaminases sériques à une insuffisance hépatocellulaire aiguë gravissime. A l'adolescence ou chez l'adulte, l'accumulation de cuivre dans le système nerveux central est responsable d'une maladie neurologique grave. Un chélateur du cuivre, la D-pénicillamine, est utilisé habituellement avec succès pour le traitement de cette maladie. Dans quelques cas cependant (insuffisance hépatocellulaire grave, rechute aiguë à l'arrêt du traitement), une transplantation hépatique est nécessaire et permet non seulement la guérison de la maladie hépatique, mais aussi le rétablissement d'un métabolisme normal du cuivre et la régression des signes neurologiques éventuellement déjà présents $[7,8]$.

- Les glycogénoses de type I et I bis, transmises sur un mode récessif autosomique, sont dues à un défaut d'activité soit de la glucose-6-phosphatase elle-même (type I), soit d'une translocase qui permet au glucose-6phosphate d'entrer dans les microsomes où il est clivé par la glucose6-phosphatase (type I bis). Le défaut fonctionnel de la glucose-6-phosphatase entraîne l'augmentation de la concentration de glycogène dans le foie, une tendance permanente aux hypoglycémies graves, une acidose lactique sévère, un retard de croissance et une hypercholestérolémie. Des adénomes hépatiques peuvent apparaître, exposant aux risques d'hémorragie intra-hépatique, d'hémopéritoine et de cancer du foie à l'adolescence ou à l'âge adulte. La transplantation hépatique corrige le déficit enzymatique et permet la restauration d'un métabolisme normal du glucose [9].

- La glycogénose de type IV, due à un déficit en enzyme "branchante », se manifeste par une cirrhose aboutissant à la mort au bout de quelques années. La transplantation hépatique a été faite chez quelques enfants atteints de cette maladie [10].

- Quatre malades atteints d'hémophilie $A$ et d'une hépatite chronique post-transfusionnelle avec cirrhose ont subi une transplantation hépatique [11]; chez les trois survivants les activités plasmatiques du facteur VIII coagulant étaient normales après la transplantation, confirmant que chez l'homme comme chez le chien, le foie (hépatocytes et/ou cellules endothéliales) est la principale source de facteur VIII.

- Maladies exposant à des complications extra-hépatiques graves. Un petit nombre de malades ont reçu une transplantation hépatique non pas en raison d'une maladie létale du foie mais parce qu'un défaut génétique d'une fonction spécifiquement ou principalement hépatique était responsable d'une atteinte sévère 
RÉFÉRENCES

1. Bismuth $\mathrm{H}$, Castaing D, Ericzon BG, Otte JB, Rolles K, Ringe B. Hepatic transplantation in Europe: first report of the European liver transplant registry. Lancet $1987 ; 2: 674-6$.

2. Esquivel CO, Iwatsuki S, Gordon RD, et al. Indications for pediatric liver transplantation. J Pediatr 1987; 111 : 1039-45.

3. Hood JM, Koep LJ, Peters RL, et al. Liver transplantation for advanced liver disease with alpha-1-antitrypsin deficiency. $N$ Engl J Med 1980; 302: 272-5.

4. Van Furth R, Kramps JA, Van Der Putten ABMM, Krom RAF, Gips $\mathrm{CH}$. Change in $\alpha$ l-antitrypsin phenotype after orthotopic liver transplant. Clin Exp Immunol 1986; 66 :66972.

5. Van Thiel DH, Gartner LM, Thorp FK, et al. Resolution of the clinical features of tyrosinemia following orthotopic liver transplantation for hepatoma.J He patol 1986; $3: 42-8$.

6. Tuchman M, Freese DK, Sharp HL, Ramnavaine MLR, Ascher N, Bloomer JR. Contribution of extra-hepatic tissues to biochemical abnormalities in hereditary tyrosinemia type I : study of three patients after liver transplantation. J Pediatr 1987; 110 399-402.

7. Sokol RJ, Francis PD, Gold SH, Ford DM Lum GM, Ambruso DR. Orthotopic liver transplantation for acute fulminant Wilson disease. J Pediatr 1985 ; 107 : 548-52.

8. Polson RJ, Rolles K, Calne RY, Williams R, Mardsen D. Reversal of severe neurological manifestations of Wilson's disease following orthotopic liver transplantation. Quart J Med 1987; 64 : 685-91.

9. Malatack JJ, Finegold DN, Iwatsuki S, et al. Liver transplantation for type I glycogen storage disease. Lancet 1983; 1 : 1073-5.

10. Greene HL, Ghisman FK, Brown B, McClenathan DT, Freese D. Hypoglycemia in type IV glycogenosis: hepatic improvemen in two patients with nutritional management. J Pediatr 1988; 112 : 55-8.

11. Bontempo FA, Lewis JH, Gorenc TJ, et al. Liver transplantation in hemophilia $A$. Blood 1987 ; 69 : 1721-4.

12. Morgan SH, Watts RWE. Perspectives dans l'exploration et le traitement de l'hyperoxalurie primitive de type I. Actualités Néphrologiques de l'Hôpital Necker. Paris Flammarion Médecine-Science, 1988: 99-108.

13. Kaufman SS, Wood RP, Shaw BW, et al. Orthotopic liver transplantation for type I Crigler-Najjar syndrome. Hepatology 1986 6: 1259-62. d'autres organes.

- L'hyperoxalurie de typeI entraînant une oxalose est caractérisée par un trouble du métabolisme de l'oxalate. La maladie touche surtout l'enfant et l'adulte jeune, et se marque par une lithiase rénale oxalocalcique, une néphrocalcinose et une insuffisance rénale. L'enzyme déficiente (Tableau I) a été localisée dans les peroxysomes hépatiques. Une transplantation hépatique combinée à une transplantation rénale, a été effectuée chez six malades, conduisant à cinq succès. La période postopératoire est marquée par la mobilisation des dépôts tissulaires d'oxalate de calcium et la restauration d'un métabolisme normal de l'oxalate [12].

- La maladie de Crigler-Najjar type I est une maladie rare transmise sur un mode récessif autosomique, due à une absence complète d'activité de la bilirubine UDP glycuronyl transférase, enzyme conjuguant la bilirubine dans l'hépatocyte. Cette activité n'est pas inductible par le phénobarbital, à la différence de ce qui est observé dans la maladie de CriglerNajjar de type II. L'accumulation dans le plasma de bilirubine non conjuguée à des concentrations extrêmement élevées peut, à tout moment, déborder les possibilités de liaison de la bilirubine par l'albumine et conduire à l'apparition dans le plasma de bilirubine non liée à l'albumine, qui peut se fixer sur les noyaux gris centraux et entraîner des lésions neurologiques graves. La survenue possible d'accidents neurologiques en dépit de la photothérapie a conduit à proposer la transplantation hépatique pour traiter cette maladie. Les quelques observations publiées font état d'une normalisation du métabolisme de la bilirubine permettant un retour à une vie normale [13, 14]

- Une enfant atteinte d'hypercholestérolémie familiale majeure liée à un défaut de synthèse et d'expression des récepteurs membranaires des $\mathrm{LDL}^{*}$ et responsable d'un athérome précoce grave a reçu une double transplantation hépatique et cardia-

* Low density lipoproteins. que [15]; le cholestérol plasmatique qui était de $25,5 \mathrm{mmol} / \mathrm{l}$ s'est abaissé à $7,65 \mathrm{mmol} / \mathrm{l}$ après la transplantation ; un traitement complémentaire par un médicament stimulant l'expression des récepteurs des LDL a permis d'obtenir la normalisation de la concentration plasmatique du cholestérol; cette normalisation se poursuit deux ans après la transplantation $[15,16]$; ceci suggère que la masse de récepteurs des LDL présents sur les cellules du foie est capable d'assurer un métabolisme normal du cholestérol en dépit de la persistance du déficit sur les autres cellules de l'organisme.

- Une publication récente rapporte la correction d'un déficit homozygote en protéine $C$, responsable de thromboses graves, par transplantation hépatique chez un enfant de 20 mois [17].

Ces résultats montrent que: (a) la transplantation hépatique peut corriger des anomalies génétiques se révélant par une maladie du foie ou par l'atteinte d'autres organes; (b) un défaut génétique potentiellement présent dans tout l'organisme peut être compensé par la masse des cellules apportées par la transplantation hépatique; (c) le pourcentage de survie après transplantation pour maladie génétique est plus élevé que pour les autres maladies du foie; les indications de la transplantation hépatique pourraient donc être étendues à d'autres maladies héréditaires du métabolisme hépatique (par exemple certains déficits partiels en ornithine-carbamyl-transférase).

\section{La transplantation de moelle osseuse}

- La greffe de moelle osseuse comme traitement des maladies héréditaires de la moelle osseuse. Il a été montré au cours des vingt dernières années que la transplantation médullaire pouvait permettre de traiter de façon efficace toutes les maladies héréditaires potentiellement létales de la moelle osseuse [18, 25]. Ceci est vrai que le déficit soit quantitatif ou qualitatif, restreint aux lignées lymphoïdes, plaquettaires, érythroïdes, myéloïdes, aux ostéoclastes ou plus global (aplasie médullaire familiale) (figure 1 , page 411). Un des exemples les plus 


\section{Tableau 1}

PRINCIPALES MALADIES MÉTABOLIOUES DE L'ENFANT QUI ONT BÉNÉFICIÉ D'UNE TRANSPLANTATION HÉPATIQUE

\begin{tabular}{|c|c|c|}
\hline Maladie & Défaut moléculaire & Conséquences pathologiques \\
\hline Déficit en $\alpha 1$-antitrypsine & $\begin{array}{l}\text { Mutation ponctuelle de la protéine } \\
\text { (phénotype Z) } \\
\text { Défaut de sécrétion par l'hépatocyte }\end{array}$ & $\begin{array}{l}\text { - Cirrhose (enfant) } \\
\text { - Emphysème pulmonaire (adulte) }\end{array}$ \\
\hline Tyrosinémie héréditaire & $\begin{array}{l}\text { Déficit en fumaryl acéto-acétate hydro- } \\
\text { lase }\end{array}$ & $\begin{array}{l}\text { - Insuffisance hépatocellulaire } \\
\text { - Cirrhose } \\
\text { - Cancer du foie }\end{array}$ \\
\hline Maladie de Wilson & $\begin{array}{l}\text { Défaut de synthèse de la céruloplas- } \\
\text { mine } \\
\text { Défaut d'excrétion biliaire du cuivre }\end{array}$ & $\begin{array}{l}\text { - Insuffisance hépatocellulaire } \\
\text { - Cirrhose } \\
\text { - Atteinte neurologique }\end{array}$ \\
\hline Glycogénose type I & Déficit en glucose-6-phosphatase & $\begin{array}{l}\text { - Hypoglycémies } \\
\text { - Retard de croissance } \\
\text { - Adénomes, hépatocarcinome }\end{array}$ \\
\hline Glycogénose type IV & $\begin{array}{l}\text { Déficit en alpha-1, } 4 \text { glycan } \\
6 \text {-glycosyl transférase }\end{array}$ & - Cirrhose \\
\hline Hémophilie A & Déficit en facteur VIII & $\begin{array}{l}\text { - Hémorragies } \\
\text { - Hépatites post-transfusionnelles } \\
\text { - SIDA }\end{array}$ \\
\hline $\begin{array}{l}\text { Hyperoxalurie type I } \\
\text { (pyridoxine-résistant)* }\end{array}$ & $\begin{array}{l}\text { Déficit en alanine-glyoxylate amino- } \\
\text { transférase peroxysomale hépatique }\end{array}$ & $\begin{array}{l}\text { - Lithiase rénale } \\
\text { - Néphrocalcinose } \\
\text { - Insuffisance rénale }\end{array}$ \\
\hline Crigler-Najjar type I & $\begin{array}{l}\text { Déficit en bilirubine UDP glycuronyl } \\
\text { transférase }\end{array}$ & $\begin{array}{l}\text { - Hyperbilirubibémie non conjuguée } \\
\text { - Ictère nucléaire }\end{array}$ \\
\hline Hypercholestérolémie familiale ${ }^{* *}$ & Absence de récepteur pour les LDL & - Athérosclérose précoce \\
\hline
\end{tabular}

* Transplantation foie et rein. ** Transplantation foie et cœur.

spectaculaires consiste en la correction de la maladie des os de marbre (ostéopétrose), c'est-à-dire la constatation de la néoformation de cavités médullaires (figure 2, page 411) après transplantation.

Environ un millier de transplantations ont été réalisées dans ces indications dans le monde. A l'heure actuelle, une transplantation effectuée à partir de donneur HLA* identique a environ huit chances sur dix de réussir. Ce pourcentage est même supérieur dans le cas des déficits immunitaires combinés sévères. La guérison, sauf rarissime exception, paraît définitive.

- Quels malades greffer et quand les greffer? Certains choix sont simples. Un enfant atteint d'un déficit immunitaire combiné sévère a une espérance de vie au plus de quelques $\mathrm{m} / \mathrm{s} n^{\circ} 7$ vol. 4 , septembre 88 mois et doit donc être greffé le plus précocement possible [25]. Parfois, le choix est ardu. L'exemple, évident pour des raisons tant médicales qu'économiques, est la $\beta$-thalassémie [21] . Le traitement de cette maladie par transfusions sanguines répétées et chélation du fer offre une espérance de vie de plusieurs dizaines d'années, sans guérison. Cependant, la greffe de moelle effectuée à partir d'un donneur compatible guérit huit malades sur $\operatorname{dix}^{* *}$, mais deux décèdent et voient donc leur espérance de vie abrégée.

- Le futur immédiat. Deux malades

\section{* Human leucocyte antigen.}

* Avec les complications possibles de la greffe de moelle: déficit immunitaire, $\mathrm{Gl} / \mathrm{H}$ chronique.

sur trois susceptibles d'être traités efficacement par transplantation médullaire n'ont pas de donneur HLA identique dans leur famille. Les quelques succès des transplantations de foie foetal réalisées dans la décennie 1970-1980, pour traiter des enfants atteints de déficits immunitaires combinés sévères, ont montré qu'il était théoriquement possible de contourner l'obstacle de l'incompatibilité entre donneur et receveur dans le système HLA. Malheureusement, ce résultat est d'importance pratique limité car observé seulement une à deux fois sur dix [26]. La greffe de moelle osseuse partiellement HLA incompatible, déplétée en lymphocytes $T$ [27] est efficace six fois sur dix comme traitement des déficits immunitaires combinés sévères [25]. Ailleurs cette méthode est inefficace car 


\section{RÉFÉRENCES}

14. Shevell MI, Bernard B, Adelson JW, Doody DP, Laberge JM, Guttman FM. Crigler-Najjar syndrome type I : treatment by home phototherapy followed by orthotopic liver transplantation. J Pediatr 1987; 110 : 429-31.

15. Bilheimer DW, Goldstein JL, GrundySM, Starzl TE, Brown MS. Liver transplantation to provide low-density lipoprotein receptors and lower plasma cholesterol in a child with homozygous familial hypercholesterolemia. N Engl J Med 1984; 311 : 1658-64.

16. East C, Grundy SM, Bilheimer DW. Normal cholesterol levels with Lovastatin (Mevinolin) therapy in a child with homozygous familial hypercholesterolemia following liver transplantation. JAMA 1986 ; 256 : 2843-8.

17. Casella JF, Lewis JM, Bontempo FA, Zitelli BJ, Markel H, Starzi TH. Successful treatment of homozygos protein $\mathrm{C}$ deficiency by hepatic transplantation. Lancet 1988; 1 : 435-8.

18. Gatti RA, Meuwissen HJ, Allen HD, et al. Immunological reconstitution of sex-linked lymphogenic immunological deficiency. Lancet $1968 ; 2$ : $1366-9$

19. O'Reilly RJ, Brochstein J, Dinsmore R, et al. Marrow transplantation for congenital disorders. Semin Hematol 1984; 21 : 188-221.

20. Parkman R. The application of bone marrow transplantation to the treatment of genetic diseases. Science 1986; 232 : 1373-8.

21. Lucarelli G, Galimberi M, Polchi P, et al. Marrow transplantation in patients with advanced thalassemia. N Engl J Med 1987 ; 316: 1050-5.

22. Gluckman E, Berger R, Dutreix A. Bone marrow transplantation for Fanconi anemia. Sem Hematol 1984; 21 : 20-6.

23. Ballet JJ, Griscelli C, Coutris C, et al. Bone marrow transplantation in osteopetrosis. Lancet $1977 ; 2$ : 1137-9.

24. Coccia PF, KrivitW, Cervenka J, et al. Successful bone marrow transplantation for infantile malignant osteopetrosis. N Engl J Med 1980; 362 : 701-8.

25. Fischer A, Griscelli C, Friedrich W, et al. Bone marrow transplantation for immunodeficiencies and osteopetrosis. An european survey 1968-1985. Lancet $1986 ; 2: 1080-4$.

26. O'Reilly R J, Kapoor N, Kirkpatrick D, et al. Transplantation of hematopoïetic cells for lethal congenital immunodeficiencies. Birth Defects 1983 ; 19 : 129-37.

27. Korngold R, Sprent J. Lethal graft-versus-host disease after bone marrow transplantation accross minor histocompatibility bar- le système immunitaire du receveur est capable dans un grand nombre de cas (40 à $90 \%$ ) [27-29] de reconnaître les antigènes HLA spécifiques du donneur et de provoquer le rejet de la greffe. Afin d'éviter ce phénomène de rejet, des méthodes d'immunosuppression du receveur à l'aide d'anticorps monoclonaux sont à l'étude. Certains essais donnent des résultats encourageants. C'est ainsi le cas de l'utilisation d'un anticorps dirigé contre une molécule d'adhésion des lymphocytes T : LFA-I ( $75 \%$ de prise, $55 \%$ de succès) (voir $\mathrm{m} / \mathrm{s} n^{\circ} 6$, vol. 3 , p. 334)

Une autre voie de recherche consiste en la sélection d'un donneur HLA compatible hors de la famille. Cette solution paraît théoriquement idéale mais se heurte à plusieurs difficultés : l'identité des antigènes HLA telle qu'elle est définie par les méthodes usuelles de typage (sérologie et culture mixte) n'implique pas forcément une identité parfaite. De plus la probabilité de différences dans d'autres systèmes polymorphes (antigènes mineurs d'histocompatibilité) est plus grande entre deux individus non apparentés. Compte tenu du polymorphisme du système HLA, la recherche d'un tel donneur nécessite le typage d'un grand nombre de sujets et une organisation bien rôdée. Une telle banque s'est constituée en France au cours de ces dernières années (voir flash de C. Raffoux).

- Le futur lointain (la génothérapie). Logiquement, l'allogreffe de moelle devrait à terme laisser la place à l'autogreffe de moelle. Il devrait en effet être possible de corriger in vitro l'anomalie génétique présente dans les cellules souches médullaires par incorporation du gène normal. Une telle approche s'avère efficace in vitro en utilisant 'un vecteur rétroviral défectif (incapable de se répliquer) et comportant le gène intéressant. De nombreux problèmes se posent encore quant à l'expression in vivo, la stabilité de celle-ci et l'innocuité d'un tel traitement (comme le montrent les tentatives d'expression du gène de l'adénosine désaminase humaine chez la souris ou le singe). Il est remarquable d'observer qu'une équipe américaine a récemment obtenu l'expression stable in vivo par les cellules médullaires de quelques souris, de la chaîne $\beta$ de l'hémoglo- bine humaine [30].

- La greffe de moelle osseuse comme traitement de maladies héréditaires dont l'expression n'est pas restreinte à la moelle osseuse. Un tel traitement repose sur deux conditions: (1) Les ou des cellules originaires de la moelle osseuse produisent la substance (l'enzyme) déficiente dans l'organisme; (2) L'enzyme et/ou le substrat de celle-ci sont largement diffusibles.

Ces deux conditions ne sont remplies que dans un petit nombre de maladies héréditaires et rendent inopérant le traitement par transplantation médullaire de maladies telles que la mucoviscidose ou les myopathies. Les maladies liées à des déficits en enzymes exprimées par des cellules hématopoïétiques sont les mucopolysaccharidoses liées à des déficits en enzymes lysosomiales, certaines mucolipidoses et sphingolipidoses comme la leucodystrophie métachromatique ou l'adrénoleucodystrophie. Lorsque la maladie n'affecte pas le système nerveux central (SNC), la guérison est effectivement possible. Ceci a été vérifié dans un exemple: la mucopolysaccharidose (MPS) de type VI «Maroteaux-Lamy » mais pas dans un autre, la MPS de type IV « Morquio » [31, 32].

L'atteinte du SNC comporte une difficulté supplémentaire: l'accessibilité du système nerveux aux cellules hématopoïétiques (monocytes essentiellement) provenant du donneur. La barrière hémoméningée est en effet peu perméable aux cellules d'origine médullaire. Expérimentalement, il a été montré par Hougerbrugge et al. [33] que des celllules du donneur n'étaient retrouvées au niveau du SNC que chez des souris atteintes de maladies génétiques s'accompagnant de lésions inflammatoires du cerveau (par exemple la souris twitcher) et non pas en leur absence (déficit en glucocérébrosidase) [33]. Chez l'homme, des cellules mononuclées de donneur ont été identifiées dans le liquide céphalorachidien après transplantation chez un patient atteint de leucodystrophie métachromatique. Toutefois, l'amélioration clinique observée en corollaire n'a été que transitoire [34]. Quelques observations de malades atteints de MPS de type I (maladie de Hurler) suggèrent que la transplan- 


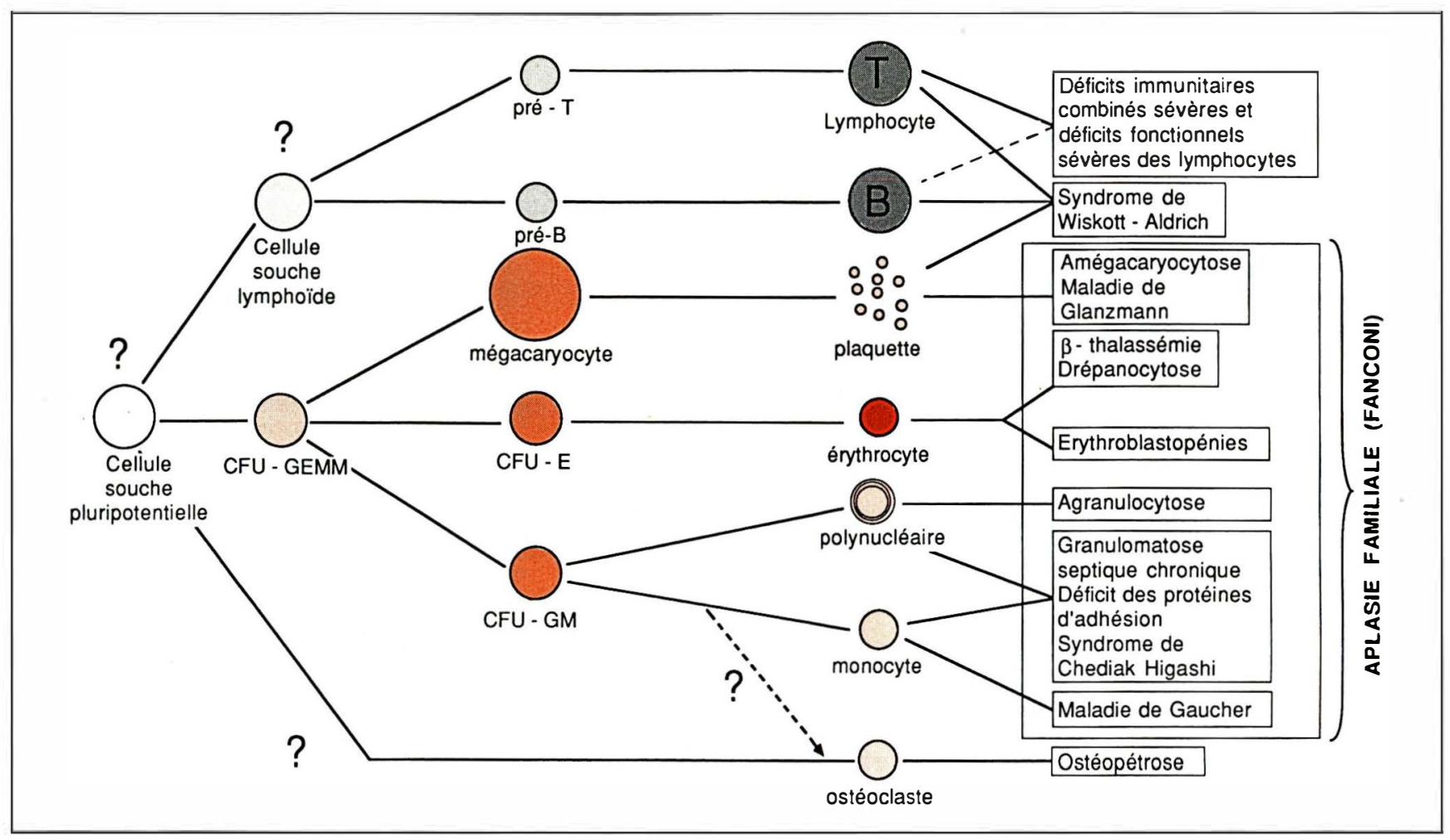

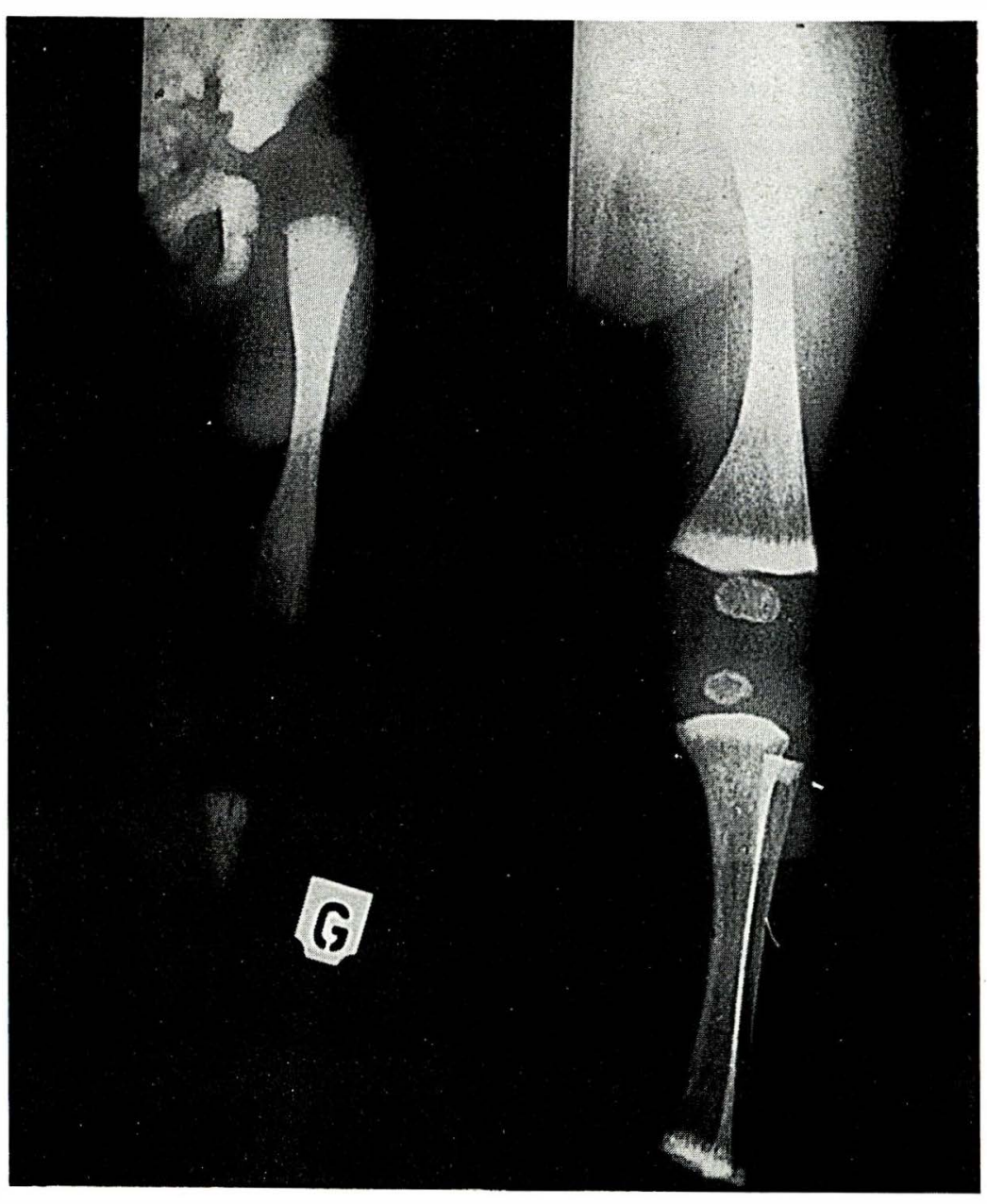

$\mathrm{m} / \mathrm{s} n^{\circ} 7$ rol. 4 , septembre 88
Figure 1. Schéma simplifié de la différenciation des principales lignées hématopoïétiques et des maladies héréditaires correspondantes traitées par allogreffe de moelle osseuse. (?) = cellule non caractérisée ; CFUGEMM = cellules donnant naissance in vitro à des colonies mixtes : granulocyte, érythrocyte, monocyte et mégacaryocyte : CFUE =cellules donnant naissance in vitro à des colonies d'érythrocytes; CFUGM = cellules donnant naissance in vitro à des colonies de granulocytes et de monocytes.

4 Figure 2. Ostéopétrose. Modification de la densité osseuse après greffe de moelle osseuse. Les radiographies montrent le membre inférieur avant greffe (à droite) et après greffe (lettre G, à gauche). 
tation médullaire permettrait d'éviter la dégradation mentale inéluctable [35, 36]. En parallèle, l'équipe de Kriwit a montré une diminution des glycosaminoglycanes dans le liquide céphalorachidien de ces malades. Ces résultats paraissent encourageants mais sont encore très limités tant dans le nombre que le recul. La plus grande circonspection est donc légitime, appuyée sur les données établies dans les modèles expérimentaux murins.

\section{La transplantation rénale}

Comme pour le foie ou la moelle osseuse, certains déficits métaboliques héréditaires, «localisés» au rein, peuvent aboutir à la destruction

\section{RÉFÉRENCES}

28. Fischer A, Griscelli C, Blanche S, et al. Prevention of graft failure by an anti LFA. 1 monoclonal antibody in HLA mismatched bone marrow transplantation. Lancet 1986; 2 : 1058-61.

29. Zwaan FE. The use of donors other than HLA-identical siblings in bone marrow transplantation. In : Gale RP, Champlin RC, eds. A workshop Summary in Progress of Bone Marrow Transplantation. New York: Alan R. Liss Inc, $1987: 677-87$

30. Dzierzak EA, Papayannopouloo T, Mulligan RC. Lineage-specific expression of a human $\beta$ globin gene in murine bone marrow transplant recipients reconstituted with retrovirus-transduced stem cells. Nature 1988 ; $331: 35-41$.

31. Kriwit W, Pierpont ME, AlazK, et al. Bone marrow transplantation in MaroteauxLamy syndrome (mucopolysaccharidosis type VI). N Engl J Med 1984; 311 : 1606-11.

32. Barranger JA. Marrow transplantation in genetic diseases. $N$ Engl J Med 1984; 311 : 1629-31.

33. Hoogerbrugge PM, Suzuki K, Suzuki K et al. Donor-derived cells in the central nervous system of Twitcher mice after bone marrow transplantation. Science 1988; 239 : 1035-8.

34. Ladisch S, Bayever E, Philipport M, et al. Biochemical findings after bone marrow transplantation for metachromatic leukodystrophy. A preliminary report. Birth defects $1986 ; 22$ : 69-78.

35. Hobbs JR. Correction of 34 genetic diseases by displacement bone marrow transplantation. Plasm Ther Transfus Technol $1985 ; 6: 221-46$

36. Kriwit W, Whitley CB. Bone marrow transplantation for genetic diseases. N Eng

\section{Summary}

Organ transplantation has been used as therapy in some inherited metabolic diseases. Liver transplantation has been performed in children with $\alpha$-1-antitrypsin deficiency (but we do not yet know whether pulmonary emphysema will be prevented in adult life), with hereditary tyrosinemia, Wilson's disease (with regression of not only hepatic but also neurologic symptoms), type I glycogenosis, hemophilia A, type I pyridoxin-resistant primary hyperoxaluria (combined with kidney transplantation to correct the metabolic defect and prevent recurrence of renal oxalosis), type I Crigler-Najjar disease, and homozygous familial hypercholesterolemia (combined with heart transplantation). Bone marrow transplantation has been used to correct severe defects of hemopoiesis such as severe immune deficiencies, WiskottAldrich syndrome, $\beta$-thalassemia, erythroblastopenias, Gaucher's disease, osteopetrosis, etc. In some cases, e.g. $\boldsymbol{\beta}$-thalassemia, the riskbenefit ratio should be carefully evaluated, because of the risk of graft-versus-host reaction. In vitro gene introduction into autologous bone marrow cells should be considered in the future. In addition, bone marrow transplantation has been successfully performed in metabolic diseases with visceral non-hematological manifestations such as some mucopolysaccharidoses and metachromatic leukodystrophy. Kidney transplantation does not restore normal $\boldsymbol{\alpha}$-galactosidase A activity (except in the transplant) in Fabry's disease. This review illustrates what has been done with human organ transplantation to correct some metabolic defects and what progress remains to be achieved in the future to extend its indications.

\section{TIRÉS A PART}

O. Bernard: service d'hépatologie, département de pédiatrie, hôpital de Bicêtre, 94275 Kremlin Bicêtre, France. 